\title{
Modulation of Single-Atom Metal Sites for Enhanced Ambient Ammonia Electrosynthesis
}

\author{
Lili Han and Huolin Xin
}

University of California-Irvine, Irvine, California, United States

The ammonia electrosynthesis under ambient conditions represents an attractive alternative to the traditional Haber-Bosch process.[1] For it, one promising strategy has been proposed, which is electrochemical $\mathrm{N}_{2}$ reduction reaction (NRR).[2] More recently, as an emerging area of heterogeneous catalysis, single-atom (SA) catalysts provide a platform for exploring new NRR catalysts as well as revealing the fundamental mechanisms of NRR.[2,3] To date, great advancement has been achieved in the development of various SA-based NRR catalysts. However, guidelines for designing efficient SA catalysts of NRR are rarely reported. In particular, previous studies have confirmed that the loading and the coordination environments of metal atoms in SA-based catalysts have great influences on their electronic structures and subsequently affect their catalytic activities.[4,5] But, rationally tuning the loading and the structures of single-atomic active sites for NRR remains an urgent task and has not been explored before.

Herein, we accomplish the modulation of single-atomic metal sites and build optimized single Mo sites anchored on porous carbon by delicately controlling metal precursor concentrations. The modulated structures were well characterized by atomic-resolution high-angle annular dark-field scanning transmission electron microscope (HAADF-STEM) imaging, energy-dispersive X-ray spectroscopy (EDS) mapping and electron energy loss spectroscopy (EELS) (Figure 1). Consequently, the NRR performance depends on the Mo loading and the Mo active site structure (Figure 2). When the Mo content was changed from 0 to $13.40 \mathrm{wt} \%$, the $\mathrm{NH}_{3}$ yield rate gradually increased at first, reached the top point at $9.54 \mathrm{wt} \%$ and then decreased. This optimization process suggests the following mechanism. Increasing the Mo content, namely the density of the atomically dispersed active sites can enhance the activation of $\mathrm{N}_{2}$ molecules and stabilization of $\mathrm{N}_{2} \mathrm{H}$ while destabilizing $\mathrm{NH}_{2}$ species.[6] But, when the Mo content is too large, we have found that Mo-related nanoclusters appear, which decreases the amount of the single Mo sites and thus worsens the NRR performance. This worsening also indicates the core role of atomically dispersed Mo atoms bonded to $\mathrm{N}$ atoms in the NRR electrocatalysis. 
a

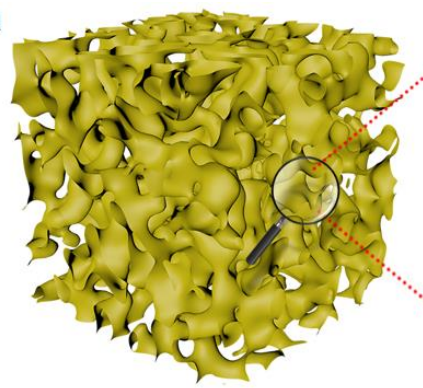

C

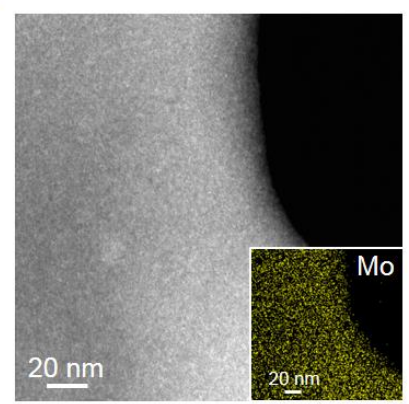

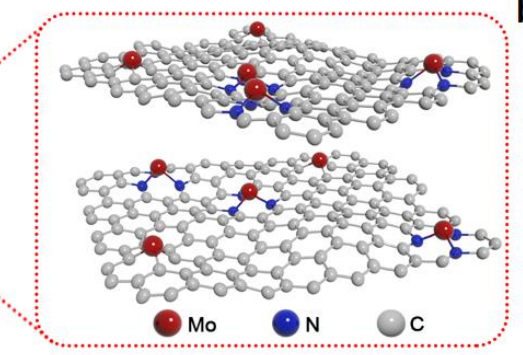

d

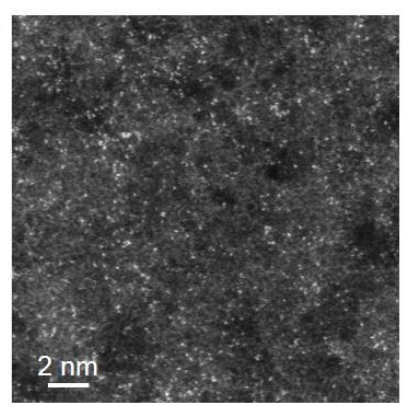

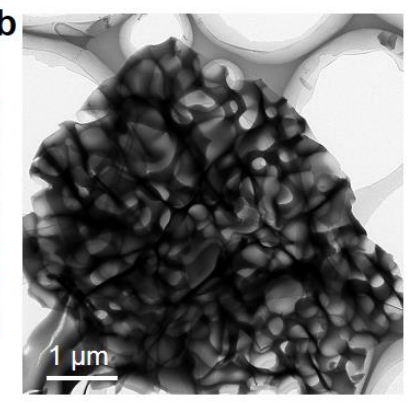

e

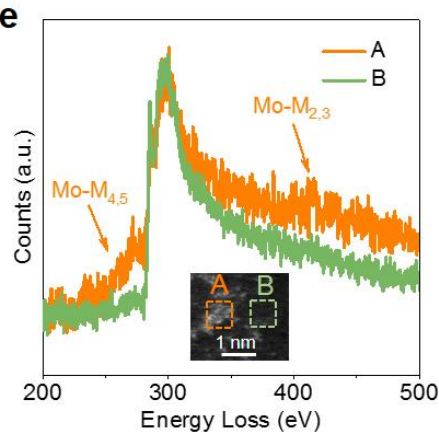

Figure 1. Structure of SA-Mo supported on N-doped porous carbon. (a) Schematic illustration and its corresponding atomic structure model. (b) TEM image. (c) HAADF-STEM image, in which the inset is the corresponding Mo EDS mapping, revealing the homogeneous distribution of Mo on the carbon support. (d) Atomic-resolution HAADF-STEM image. (e) EELS spectra from areas A and B in the atomicresolution HAADF-STEM image of the inset, in which areas A and B do not contain single Mo atoms, respectively. The two orange arrows point to the signals of Mo M4,5 and L2,3 edges, respectively.

a

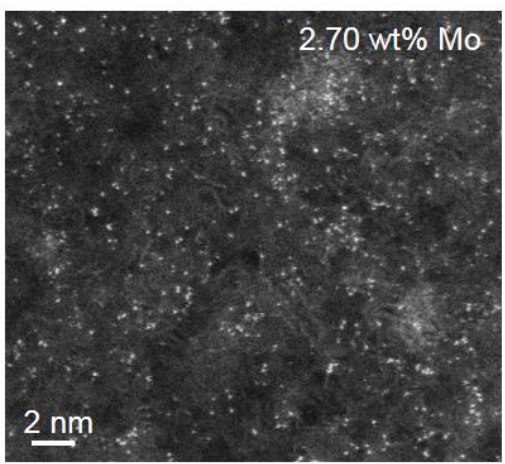

C

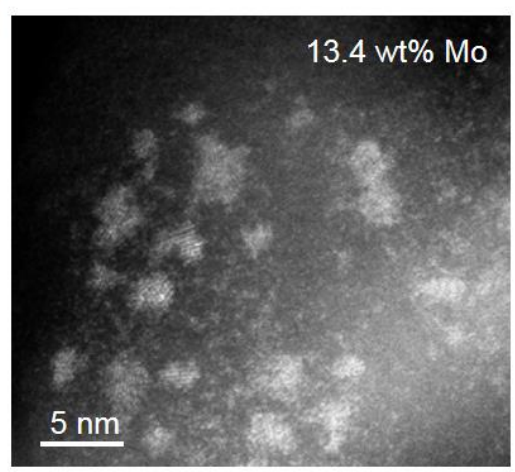

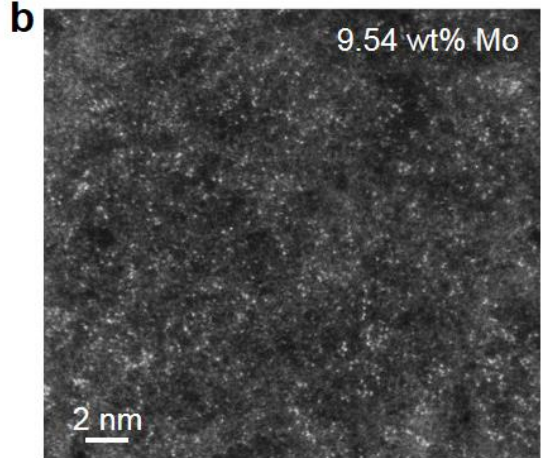

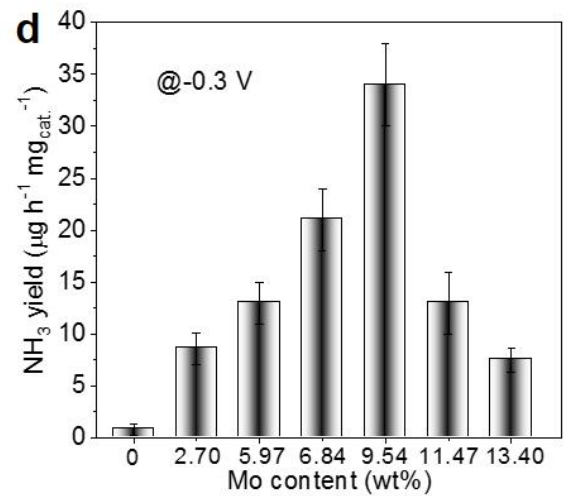


Figure 2. Atomic-resolution HAADF-STEM images and NH3 yield rates of SA-Mo supported on Ndoped porous carbon with different Mo loadings. (a) $2.7 \mathrm{wt} \% \mathrm{Mo}$, (b) $9.54 \mathrm{wt} \% \mathrm{Mo}$, (c) $13.4 \mathrm{wt} \% \mathrm{Mo}$, (d) NH3 yield rates of SA-Mo supported on N-doped porous carbon with different Mo loadings.

\section{References}

[1] Hao, Y.-C., Guo, Y., Chen, L.-W., Shu, M., Wang, X.-Y., Bu, T.-A., Gao, W.-Y., Zhang, N., Su, X., Feng, X., Zhou, J.-W., Wang, B., Hu, C.-W., Yin, A.-X., Si, R., Zhang, Y.-W \& Yan, C.-H. Nat. Catal. 2, 448-456 (2019). [2] Han, L., Liu, X., Chen, J., Lin, R., Liu, H., Lu, F., Bak, S., Liang, Z., Zhao, S., Stavitski, E., Luo, J., Adzic, R. R. \& Xin, H. L. Angew. Chem. Int. Ed. 58, 2321-2325 (2019). [3] Zhang, L., Han, L., Liu, H., Liu, X. \& Luo, J. Potential-Cycling Synthesis of Single Platinum Atoms for Efficient Hydrogen Evolution in Neutral Media. Angew. Chem. Int. Ed. 56, 13694-13698 (2017). [4] Pan, Y., Chen, Y., Wu, K., Chen, Z., Liu, S., Cao, X., Cheong, W.-C., Meng, T., Luo, J., Zheng, L., Liu, C., Wang, D., Peng, Q., Li, J. \& Chen, C. Nat. Commun. 10, 4290 (2019). [5] Yin, X.-P., Wang, H.-J., Tang, S.-F., Lu, X.-L., Shu, M., Si, R. \& Lu, T.-B. Angew. Chem. Int. Ed. 57, 9382-9386 (2018). [6] Zhao J. \& Chen Z. J. Am. Chem. Soc. 139, 12480-12487 (2017). 